J. Clin. Chem. Clin. Biochem.

Vol. 20, 1982, pp. 39-47

\title{
Untersuchungen zur Analytik von Propafenon mittels direkter Isotopenverdünnung
}

\author{
Von E. Brode \\ Aus der Experimentell-Medizinischen Forschung und Entwicklung, Department Biochemie, Unternehmensbereich \\ Pharma, BASF Aktiengesellschaft, Ludwigshafen am Rhein
}

(Eingegangen am 27. August 1981)

Zusammenfassung: Zur Bestimmung von Propafenon in Plasma wird ein hochdruckflüssigkeitschromatographisches Verfahren nach dem Prinzip der direkten Isotopenverdünnung beschrieben.

Bei Einsatz von $2 \mathrm{ml}$ Probe und Quantifizierung über Peakhöhenmessung liegt die untere Nachweisgrenze bei etwa $2,5 \mu \mathrm{g} / \mathrm{l}$, die untere Bestimmungsgrenze bei etwa $10 \mu \mathrm{g} / \mathrm{l}$. Im Konzentrationsbereich bis $\mathrm{zu} 250 \mu \mathrm{g} / 1$ ist mit einem mittleren gewichteten Relativfehler von $3,5 \%$ zu rechnen.

Bei Konzentrationen oberhalb von $20 \mu \mathrm{g} / \mathrm{l}$ erweist sich diese Methode bezüglich Richtigkeit und Präzision dem Verfahren mit interner Analogstandardisierung als gleichwertig.

Sie bietet die Möglichkeit, durch interne Standardisierung jeder einzelnen Probe unmittelbar nach ihrer Gewinnung von eventuellen Lagerverlusten unabhängig zu werden.

\section{Determination of propafenone in plasma by HPLC with direct isotope dilution}

Summary: A high performance liquid chromatography method based on the principle of direct isotope dilution is described for the determination of propafenone in plasma.

With a sample volume of $2 \mathrm{ml}$ and quantification by peak height measurements the lower limit of detection is about $2.5 \mu \mathrm{g} / \mathrm{l}$, and the lower limit of determination about $10 \mu \mathrm{g} / \mathrm{l}$. Values up to $250 \mu \mathrm{g} / \mathrm{l}$ are subject to a mean weighted relative error of $3.5 \%$.

At concentrations above $20 \mu \mathrm{g} / \mathrm{l}$ this method shows the same accuracy, and reproducibility as the analytical procedure employing internal standardization with analogues.

The method is made independent of possible storage losses by internal standardization of each sample immediately after withdrawal.

\section{Problemstellung}

Stand der Technik bei der Bestimmung der Konzentration von Wirkstoffen in komplexen biologischen Matrices sind heute intern standardisierte Analysenmethoden, die es erlaubèn, durch Vorschaltung aufwendiger, in ihren Ausbeuten variabler Trenn- oder Derivatisierungsschritte die notwendige analytische Selektivität zu erreichen, die vor allem bei stark verstoffwechselten Substanzen ein Problem darstellt.

Als interne Standards bieten sich einmal Analoge der zu bestimmenden Substanz, die möglichst ähnliche physikalisch-chemische und chemische Eigenschaften haben sollten, aber dennoch getrennt quantifizizierbar sein müssen, zum anderen die mit Isotopen markierte Substanz selbst an.
Die interne Analogstandardisierung $(1,2)$ wird heute bereits vielfältig angewendet; sie hat jedoch ihre Schwierigkeiten bei der Auswahl des geeigneten Standards; darüberhinaus ișt ihre Störanfälligkeit gegenüber ungewöhnlichen und deshalb bei der Prüfung der Methode nicht berücksichtigten Bestandteilen der zu untersuchenden Patientenplasmen sowie gegenüber gleichzeitig verabreichten Medikamenten und deren Metaboliten prinzipiell doppelt so groß wie bei einer Standardisierung mit einer markierten Substanz: die Richtigkeit der Analyse ist nur dann gegeben, wenn nach dem Trennungsschrittt Substanz und Standard frei von störenden Verunreinigungen sind.

Die Eigenstandardisierung mit markierter Substanz spielt im Vergleich zur Analogstandardisierung 
hinsichtlich ihrer Anwendungshäufigkeit nur dann eine Rolle, wenn es sich dabei um ein Analysenverfahren handelt, bei dem markierte und unmarkierte Substanz unabhängig voneinander quantitativ bestimmt werden können. Dies ist der Fall bei stabilisotopenmarkierten internen Standards, die mit massenspektrometrischer Detektion neben den entsprechenden unmarkierten Verbindungen empfindlich, präzise und richtig quantifiziert werden können (3-9). In dieser Version arbeitet die Eigenstandardisierung mit einer ähnlichen Fehlerstruktur wie die Analogstandardisierung, die Fortpflanzung der experimentellen Fehler bei den eigentlichen Messungen in das Endergebnis ist völlig unproblematisch.

Anders sind die Verhältnisse bei der sogenannten direkten Isotopenverdünnung (10), wo die unmarkierte Verbindung nur als Summe gemeinsam mit dem isotopenmarkierten Standard bestimmbar ist. Hierbei treten 2 Nachteile entscheidend zutage. Einmal macht sich die Fehlerfortpflanzung besonders dann ungünstig bemerkbar, wenn wegen nicht ausreichend hoher spezifischer Radioaktivität des Standards oder einer sehr geringen zu bestimmenden Konzentration das Verhältnis von Substanz zu Standard nicht genügend hoch ist. Zum zweiten muß eine ausreichend empfindliche Methode zur Verfügung stehen, da hier ja nicht - wie bei den sogenannten inversen Isotopenverdünnungsmethoden (10) durch Zugabe inaktiven Carriers die zu bestimmenden Konzentrationen an den optimalen Arbeitsbereich der Methode angepaßt werden können.

Seit der Entwicklung hochempfindlicher Detektoren für die Chromatographie sollte diese Einschränkung jedoch keine Rolle mehr spielen; offenbar jedoch wird die allgemeine Anwendung einer solchen Methode noch durch Betrachtung der Fehlermöglichkeiten eingeschränkt.

Wir hielten es deshalb für wichtig, anhand eines Beispiels unter heutzutage zu erwartenden Randbedingungen bezüglich der zu erreichenden Bestimmungsgrenze und der zur Verfügung stehenden spezifischen Aktivität des Standards zu prüfen, wie leistungsfähig eine nach dem Prinzip der direkten Isotopenverdünnung arbeitende Methode ist.

Bei befriedigendem Ausfall dieser Prüfung würden unter Umständen zeitaufwendige Arbeiten zur Suche geeigneter Analogstandards entfallen, es böte sich außerdem die Gelegenheit, echte probeninterne Standardisierungen vorzunehmen, womit man von eventuellen Verlusten der zu bestimmenden Substanzen bei der Lagerung in biologischem Material unabhängig werden könnte.

In der vorliegenden Arbeit soll über die Ergebnisse der Prüfung einer Bestimmungsmethode mit direkter Isotopenverdünnung berichtet werden. Als Beispiel wurde das Propafenon, der Wirkstoff, der besonders bei der Behandlung von ventrikulären Tachykardien und Tachy- arrhythmien bewährten Rytmonorm ${ }^{\circledR}$-Spezialitäten ${ }^{1}$ ), gewählt, da von dieser Substanz ein ${ }^{14} \mathrm{C}$-markiertes Produkt mit einer spezifischen Aktivität von $648 \pm 7,4$ $\mathrm{MBq} / \mathrm{mmol}$ zur Verfügung steht und außerdem ein leistungsfähiges Trennverfăanren mittels HPLC (11) vorliegt, das für die Bestimmung von Propafenon mittels interner Analogstandardisierung eingesetzt wird. Damit sind die Voraussetzungen erfüllt, um einen Vergleich der direkten Isotopenverdünnung und der Analogitandardisierung unter weitgehend identischen Bedingungen anzustellen.

\section{Material und Methoden}

\subsection{Substanzen}

\section{Propafenon}

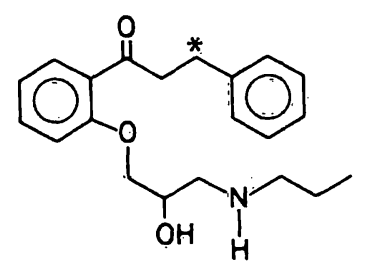

2'-(2-Hydroxy-3-propylamino-propoxy)-3-phenyl-propiophenonhydrochlorid (Charge Nr. W 34997).

${ }^{14}$ C-Propafenon (Li 838/1):

$2^{\prime}$-(2-Hydroxy-3-propylamino-propoxy)-3-phenyl- $\left[3={ }^{14} \mathrm{C}\right]$ propiophenon-hydrochlorid.

${ }^{14} \mathrm{C}$-Markierung: an der im Formelbild mit * gekennzeichneten Stelle

Hersteller:

H. Lietz, Isotopenlabor des Departments Biochemie, Unternehmensbereich Pharma BASF Aktiengesellschaft

(Datenblatt $\left[{ }^{14} \mathrm{C}\right]$-Propafenon vom 09. 01. 79)

Spez. Radioaktivität: $\quad 648 \pm 7,4 \mathrm{MBq} / \mathrm{mmol}\left(\overline{\mathrm{x}} \pm s_{\overline{\mathrm{x}}}\right)$ $(17,2 \pm 0,2 \mathrm{mCi} / \mathrm{mmol})$

Radiochem. Reinheit: nach Radiodünnschichtchromatographie $>98 \%$

Alle anderen verwendeten Chemikalien werden in der besten kommerziell verfügbaren Qualität eingesetzt.

Humanplasma wird von den Blutbanken der Städtischen Krankenanstalten Mannheim und Ludwigshafen bezogen.

2.2 Herstellung der zu analysierenden Proben und Ermittlung der Sollkonzentrationen

Aus einer Stammlösung mit $400 \mathrm{mg} / \mathrm{l}$ Propafenon wird durch Verdünnen mit Humanplasma die in den entsprechenden Tabellen aufgeführte, durch Wägung kontrollierte Konzentrationsreihe hergestellt. Die gemäß Wägungsergebnis korrigierten Konzentrationen werden den folgenden Rechnungen als Vorgabekonzentrationen zugrundegelegt.

Die so hergestellten Humanplasmaproben werden in Aliquots von je $2,0 \mathrm{ml}$ aufgeteilt und nach Zugabe von je $12,0 \mathrm{ng}{ }^{14} \mathrm{C}$ Propafenon bis zur Analyse bei $<-25^{\circ} \mathrm{C}$ tiefgefroren.

1) Im Handel als Rytmonorm ${ }^{\circledR} 150 \mathrm{mg}$, Rytmonorm $^{\circledR} 300 \mathrm{mg}$ und Rytmonorm ${ }^{\circledR}$ Ampullen; Hersteller: Knoll AG, Ludwigshafen. 


\subsection{Hochdruckflüssigkeitschrom atographie}

\subsubsection{HPLC-System}

Das eingesetzte Hochdruckflüssigkeitschromatographie-System besteht aus

dem Probenaufgabesystem Waters ${ }^{\circledR}$ U6K, der Pumpeneinheit Waters ${ }^{\circledR}$ Typ 6000A, der HPLC-Säule Merck-Hibar ${ }^{\circledR}(250 \times 4 \mathrm{~mm})$ und dem UV-Detektor Waters ${ }^{\circledR}$ Modell 440.

\subsubsection{HPLC-Bedingungen}

$\begin{array}{llrl}\text { Laufmittelsystem: } & n \text {-Heptan } & 240 & \text { Volumenteile } \\ & \text { Dichlormethan } & 360 & \text { Volumenteile } \\ & \text { Ethanol } & 700 \text { Volumenteile } \\ & \mathrm{H}_{2} \mathrm{O} & 54 \text { Volumenteile } \\ & \mathrm{NH}_{3} \text { konz. } & 0.5 \text { Volumenteile }\end{array}$

Die Laufmittelkomponenten werden gemischt, in einem Druckfiltrationsgerät (Millipore ${ }^{\circledR}$ Typ 04740) durch ein Teflonfilter (Typ Fluorophor) filtriert und anschließend entgast.

Flußrate: $2.2 \mathrm{ml} / \mathrm{min}$ bei ctwa $28-32 \mathrm{MPa}$

Trägermaterial: Si $60 / 5 \mu \mathrm{m}$ (Merck)

UV-Detektion: $254 \mathrm{~nm}$

\subsection{Messung der Radioaktivität}

Alle Messungen erfolgen nach Zugabe von $18 \mathrm{ml}$ Monophase ${ }^{\circledR}\left(F_{a}\right.$. Packard) in einem Flüssigkeitsszintillationszähler (Tri Carb ${ }^{\circledR}$, Modell 3380; Tri Carb ${ }^{\circledR}$, Modell 460 C, der Firma Packard) mit externer Standardisierung. Die Zähldauer wird durch Vorgabe eines statistischen Zählfehlers, hier $0.2 \%$, begrenzt bzw. beträgt höchstens $20 \mathrm{~min}$. Die so gemessenen Zählraten werden um den jeweiligen Nulleffekt korrigiert.

Die Quenchkorrektur erfolgt über eine den Zählausbeuten einer Standardquenchreihe rechnerisch angepaßte neunfache kubische Ausgleichssplinefunktion (WANG 2200).

\subsection{Durchführung der Methode}

$2 \mathrm{ml}$ Human plasma werden in einem Zentrifugenglas geeigneter Größe mit Schliffstopfen mit $0.1 \mathrm{ml} 1 \mathrm{~mol} / 1$ Natronlauge alkalisch gestellt. Anschließend wird $2 \mathrm{mal} \mathrm{mit} \mathrm{je} 4 \mathrm{ml} \mathrm{Benzol}^{2}$ ) extrahiert ( 1 min Intensiv-Rüttelung auf Circomix ${ }^{\circledR}$ und $15 \mathrm{~min}$ auf einem Heidolph-Laborschüttler, danach zentrifugieren und organische Phase abheben). Die organischen Phasen werden in einem konisch zulaufenden Einenggefäß unter sanftem Stickstoffstrom (hochreiner Stickstoff) bei Temperaturen unterhalb $40^{\circ} \mathrm{C}$ zur Trockne gebracht. Nach Wiederaufnahme mit $100 \mu \mathrm{l}$ Methanol wird anderentags (Aufbewahrung im Kühlschrank) analysiert, wobei etwa $80 \mu \mathrm{l}$ in das HPLC-System injiziert werden.

Das dem Propafenonpeak entsprechende Eluat wird in Scintillationsmeßgläschen aufgefangen und der Radioaktivitätsmessung zugefuhrt.

\subsection{Datenregistrierung und -reduktion}

Das Chromatogramm wird zur Datensicherung mittels Schreiber registriert und die Propafenon umfassende Region gleichzeitig digitalisiert auf Magnetband-Kassette gespeichert (A/D-

Wandler, Typ BASF-TKL, mit Kassettenstation MFE 5450 Fa. Fey, München). Die Berechnung relevanter chromatographischer Parameter (Retentionszeiten, Peakhöhen und Peakflächen) erfolgt mit Hilfe des GC-Auswerteprogramms von Caesar \& Klier (12) in einer auf die Bedürfnisse einer internen Standardisierung zugeschnittenen Version auf dem HoneywellBull-Rechner (BASF-Rechenzentrum) über Terminal.

\footnotetext{
2) Versuche, Benzol durch Toluol zu ersetzen, ergaben neben geringeren Extraktionsausbeuten auch schlechtere Reproduzierbarkeit.
}

\subsection{Auswertung}

Die Auswertung erfolgt über die Verhältnisse der Peakhöhen bzw. Peakflächen zur ${ }^{14} \mathrm{C}$-Zerfallsrate nach der Formel

$$
\begin{aligned}
c_{\mathrm{P}} & =\frac{\mathrm{m}_{\mathrm{P}}}{\mathrm{V}}=\frac{\mathrm{m}_{\mathrm{St}}}{\mathrm{V}} \times\left(\frac{\mathrm{Q}}{\mathrm{Q}_{0}}-1\right) \\
\text { mit } \quad c_{\mathrm{P}} & =\text { Propafenon-Konzentration } \\
\mathrm{m}_{\mathrm{P}} & =\text { Menge Propafenon im Ansatz } \\
\mathrm{V} & =\text { Probevolumen } \\
\mathrm{m}_{\mathrm{St}} & =\text { Menge interner Stand ard } \\
\mathrm{Q} & =\text { Verhältnis von Peakhöhen bzw. Peakflächen zu } \\
\mathrm{Q}_{0} & =\mathrm{Q} \text { für } \mathrm{m}_{\mathrm{P}}=0
\end{aligned}
$$

Der Quotient $Q_{0}$ wird für jede Analysenserie aus 2 in Duplikaten mitgeführten Kalibrierungsproben unterschiedlicher Konzentration aus der Steigung einer graphischen Darstellung berechnet, in der $Q$ als Funktion von $\left(m_{P}+m_{S t}\right)$ aufgetragen ist.

Die Zusammenfassung und Protokollierung der Daten sowie die Berechnung der verschiedenen statistischen Parameter und Tests aus den gewonnenen experimentellen Daten werden mit Hilfe einer Wang 2200 durchgeführt.

Zur statistischen Absicherung der zu diskutierenden Ergebnisse werden

per t-Test (13) ein Soll/Ist-Vergleich zur Überprüfung der Wiederfindungsquoten,

die Prüfung des Regressionskoeffizienten auf Abweichung von 1 und

- die Prüfung des Ordinatenabschnitts auf Abweichung von 0

durchgeführt (Sicherheitswahrscheinlichkeit 99\%).

Regressions- und Korrelationsanalysen erfolgen gemäl3 allgemeinen Vorschriften (14).

Zur Überprüfung der Richtigkeit und Präzision der Methode werden die Mittelwerte und deren Standardfehler für jede Konzentration in allen Analysenserien gebildet. Bei der Quotientenund Differenzbildung zur Ermittlung der Richtigkeit werden die Standardfehler in das Ergebnis statistisch fortgepflanzt.

\section{Ergebnisse und Diskussion}

Die hier geprüfte Methode ist bezüglich der Probenaufbereitung und des HPLC-T rennschritts identisch mit dem früher beschriebenen Verfahren mit interner Analogstandardisierung (11). Die dort zur Eignung der chromatographischen Bedingungen und zur Selektivität gemachten Ausführungen sind deshalb ohne weiteres auf diese Methode übertragbar.

Die Frage, ob Peakhöhe oder Peakfläche den geeigneteren Parameter für das Mengenmaß darstellt, soll erst anhand der hier erhobenen Befunde beantwortet werden.

Um die Leistungsfähigkeit dieser nach dem Prinzip der direkten Isotopenverdünnung arbeitenden Methode unter Routinebedingungen zu überprüfen, werden jeweils in Duplikaten eine Reihe von Plasmaproben mit unterschiedlichen bekannten Propafenon-Konzentrationen zwischen etwa 10 und $200 \mu \mathrm{g} / 1$ innerhalb eines Zeitraumes von 6 Wochen der oben detailliert beschriebenen Methode unterworfen. 


\subsection{Peakflächen als Meßparameter}

\subsubsection{Wiederfindung}

Angaben zur Wiederfindung von zugesetztem Propafenon nach Durchlaufen der Analysenprozedur, die bei gegebener Selektivität ein Maß für die Richtigkeit der Analysen darstellen, sind in Tabelle 1 zusammengestellt.

Im Konzentrationsbereich zwischen 11 und $220 \mu \mathrm{g} / 1$ kommt es danach zu Wiederfindungsquoten zwischen 96,6 und $101,5 \%$, die in keinem Fall signifikant von $100 \%$ abweichen. Die in der Tendenz Minderbefunde anzeigenden Werte sind möglicherweise auf eine zu gering angesetzte spezifische Aktivität (Radiolyse?) des zur Standardisierung verwendeten ${ }^{14} \mathrm{C}$-markierten Materials zurückzuführen.

Wie eine Korrelationsanalyse zwischen gegebenen und gefundenen Werten (Einzeldaten) zeigt, liefert das Analysenverfahren mit der gewählten Standardisierung richtige Ergebnisse: der Regressionskoeffizient $b=1,011 \pm 0,010$ ist nämlich nicht von 1 verschieden $(r=0,995)$.

Wie sich dies bei der Proportionalität zwischen den verschiedenen in der gleichen Analysenserie bearbeiteten Konzentrationen ausdrückt, verdeutlicht Abbildung la und $1 \mathrm{~b}$ für die Serien mit den höchsten und niedrigsten Korrelationskoeffizienten. Zur Gewährleistung der Richtigkeit erscheint es zumindest für die in Abbildung $1 \mathrm{~b}$ dargestellte Serie unerläßlich, Duplikate zu analysieren.

\subsubsection{Präzision}

Für jede der vorgegebenen Konzentrationen sind Angaben zur Präzision des Gesamtverfahrens in Tabelle 1 enthalten. Daraus kann entnommen werden, daß bei den beiden

Abb. 1a und 1b. Propafenon-Methodenprüfung (direkte Isotopenverdünnung mit Peakflächenmessung).

a) Soll/Ist-Vergleich Serie $9(r=0,999)$

b) Soll/lst-Vergleich Serie $10(r=0.985)$
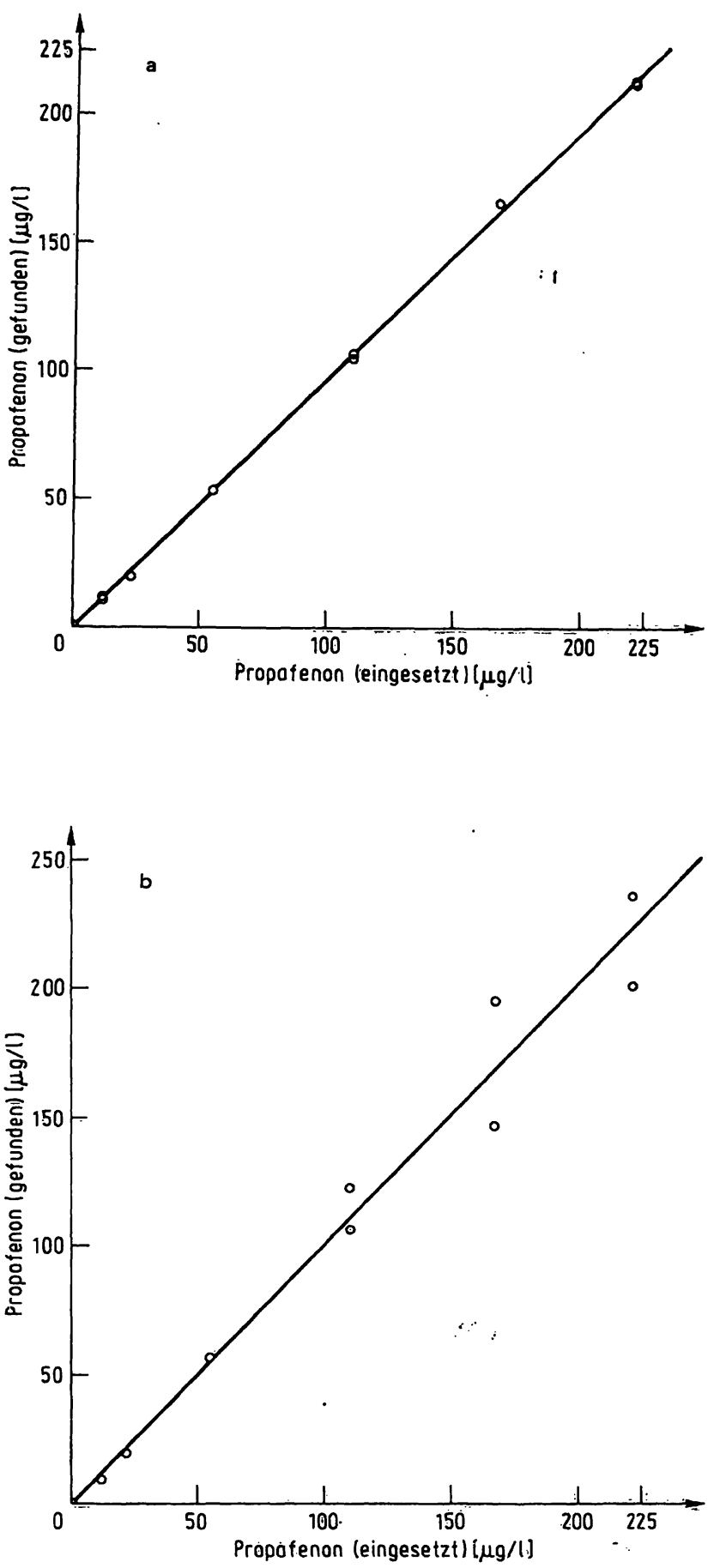

Tab. 1. Propafenon-Methodenprüfung (direkte Isotopenverdünnung mit Peakflächenmessung). Angaben zur Richtigkeit und Präzision; Mittelwert der Duplikatanalysen als Meßwert.

\begin{tabular}{|c|c|c|c|c|c|c|}
\hline \multirow[b]{2}{*}{$\begin{array}{l}\text { Vorgabe- } \\
\text { konzentration } \\
\text { gegeben } \\
(\mu \mathrm{g} / 1)\end{array}$} & \multirow[b]{2}{*}{$\begin{array}{l}\text { Mittelwert } \\
\text { gefunden } \\
(\mu \mathrm{g} / 1)\end{array}$} & \multirow[b]{2}{*}{$\mathrm{n}$} & \multirow{2}{*}{$\begin{array}{l}\text { Riçhtigkeit } \\
\text { gefunden / gegeben } \\
\text { (\%) }\end{array}$} & \multirow[b]{2}{*}{$\begin{array}{l}\text { gefunden - gegeben } \\
(\mu \mathrm{g} / 1)\end{array}$} & \multicolumn{2}{|c|}{ Präzisiọn der Methode } \\
\hline & & & & & $\begin{array}{l}\text { Standard- } \\
\text { abwei- } \\
\text { chung } \\
(\mu \mathrm{g} / 1)\end{array}$ & $\begin{array}{l}\text { Variations- } \\
\text { koeffi- } \\
\text { zient } \\
(\%)\end{array}$ \\
\hline $\begin{array}{r}11,17 \\
22,34 \\
55,53 \\
110,10 \\
167,51 \\
221,38\end{array}$ & $\begin{array}{r}10,86 \pm 0,45 \\
21,59 \pm 0,93 \\
54,10 \pm 1,06 \\
110,28 \pm 1,99 \\
167,20 \pm 2,33 \\
224,53 \pm 5,30\end{array}$ & $\begin{array}{l}8 \\
8 \\
8 \\
8 \\
8 \\
8\end{array}$ & $\begin{array}{r}97,25 \pm 4,00 \\
96,62 \pm 4,15 \\
97,41 \pm 1,91 \\
100,16 \pm 1,80 \\
99,81 \pm 1,39 \\
101,42 \pm 2,39\end{array}$ & $\begin{array}{r}-0,30 \pm 0,45 \\
-0,74 \pm 0 ; 93 \\
-1,42 \pm 1,06 \\
0,18 \pm 1,99 \\
-0,30 \pm 2,33 \\
3,15 \pm 5,30\end{array}$ & $\begin{array}{r}1,26 \\
2,63 \\
3,00 \\
5,63 \\
6,59 \\
15,00\end{array}$ & $\begin{array}{r}11,63 \\
12,17 \\
\because 5,55 \\
5,10 \\
3,94 \\
6,68\end{array}$ \\
\hline
\end{tabular}

J. Clin. Chem. Clin. Biochem. / Vol. 20,1982 / No. I 
niedrigen Konzentrationen von $\sim 11$ und $\sim 22 \mu \mathrm{g} / 1$ Variationskoeffizienten zwischen 10 und $15 \%$ erreicht werden, während darüber mit Variationskoeffizienten von etwa 4-7\% zu rechnen ist.

Die Reproduzierbarkeit des geprüften Verfahrens von Tag zu Tag ist, wie Tabelle 2 zeigt, sowohl bei Analyse von Unikaten wie auch von Duplikaten im gesamten Konzentrationsbereich nahezu identisch mit der des Gesamtverfahrens (s. oben).

Die Präzision der Doppelbestimmungen in Abhängigkeit von der Sollkonzentration ist in Tabelle 3 verzeichnet. Die durchschnittlichen Variationskoeffizienten fallen von $\sim 7,5 \%$ bei $11 \mu \mathrm{g} / \mathrm{l}$ auf schließlich $\sim 2-4 \%$ bei den höheren Konzentrationen. In etwa $10 \%$ aller Fälle ist mit Variationskoeffizienten von $>15 \% \mathrm{zu}$ rechnen.

Tab. 2. Propafenon-Methodenprüfung (direkte Isotopenverdünnung mit Peakflächenmessung). Interserienstreuung.

\begin{tabular}{|c|c|c|c|c|}
\hline \multirow{3}{*}{$\begin{array}{l}\text { Vorgabe- } \\
\text { konzentra- } \\
\text { tion } \\
(\mu \mathrm{g} / 1)\end{array}$} & \multicolumn{2}{|c|}{$\begin{array}{l}\text { Variations- } \\
\text { koeffizienten } \\
\text { bei Analysen } \\
\text { von Unikaten }\end{array}$} & \multirow[b]{3}{*}{$\overline{\mathrm{VK}}(\%)$} & \multirow[t]{2}{*}{$\begin{array}{l}\text { Variations- } \\
\text { koeffizienten } \\
\text { bei Analysen } \\
\text { von Duplikaten }\end{array}$} \\
\hline & $\begin{array}{l}\text { 1. Wert } \\
\text { 2. Wert }\end{array}$ & & & \\
\hline & VK $(\%)$ & $\mathrm{n}$ & & VK $(\%)$ \\
\hline 11,1700 & $\begin{array}{l}14,86 \\
11,87\end{array}$ & $\begin{array}{l}8 \\
7\end{array}$ & 13,47 & 11,63 \\
\hline 22,3400 & $\begin{array}{r}13,08 \\
9,95\end{array}$ & $\begin{array}{l}8 \\
6\end{array}$ & 11.74 & 12,17 \\
\hline 55,5300 & $\begin{array}{l}7.46 \\
4.83\end{array}$ & $\begin{array}{l}8 \\
8\end{array}$ & 6,14 & 5,55 \\
\hline 110,1000 & $\begin{array}{l}7,43 \\
4,37\end{array}$ & $\begin{array}{l}8 \\
8\end{array}$ & $5,90^{\circ}$ & 5,10 \\
\hline 167.5100 & $\begin{array}{l}5,63 \\
7,68\end{array}$ & $\begin{array}{l}8 \\
7\end{array}$ & 6,59 & 3,94 \\
\hline 221.3800 & $\begin{array}{l}8,05 \\
6.90\end{array}$ & $\begin{array}{l}8 \\
8\end{array}$ & 7,48 & 6,68 \\
\hline
\end{tabular}

Tab. 3. Propafenon-Methodenprüfung (direkte Isotopenverdünnung mit Peakflächenmessung). Präzision von Duplikaten (Variationskoeffizienten in \%).

\begin{tabular}{lrrrrrrr}
\hline $\begin{array}{l}\text { Vorgabekonzentra- } \\
\text { tionen }(\mu \mathrm{g} / \mathrm{l})\end{array}$ & 11,2 & 22,3 & 55,5 & 110,1 & 167,5 & 221,4 \\
\hline Serie 1 & 6,1 & 5,1 & 0,4 & 1,4 & 4,3 & 1,4 \\
Serie 2 & 5,9 & 1,5 & 1,2 & 5,5 & 2,3 & 3,4 \\
Serie 3 & 5,2 & 1,2 & 0,7 & 1,1 & 0,4 & 0,8 \\
Serie 4 & 3,2 & $\overline{0}$ & 0,8 & 2,7 & 0,2 & 2,9 \\
Serie 5 & 15,5 & 16,9 & 5,7 & 0,2 & - & 1,1 \\
Serie 6 & 19,1 & 5,1 & 9,8 & 3,8 & 1,1 & 5,0 \\
Serie 7 & 4,9 & 2,0 & 0,0 & 0,9 & 0,2 & 0,6 \\
Serie 8 & - & - & 0,6 & 9,7 & 19,9 & 14,4 \\
& & & & & & \\
Mittelwert: & 7,5 & 4,0 & 2,4 & 3,2 & 3,5 & 3,7 \\
\hline
\end{tabular}

-: keine Duplikate vorhanden.
Wenn man bei der Anwendung der Methode im Routinebetrieb gerade noch Variationskoeffizienten von 15\% bei den Doppelbestimmungen akzeptiert - das entspricht einem Verhältnis von 1:1,25 bei den Einzelbestimmungen -, so hat man zu erwarten, daß etwa $10 \%$ der Analysen wiederholt werden müssen.

Wie Abbildung 2, in der die Meßfehler (Standardabweichungen unter Verwendung der Duplikateinzelwerte; s) in Abhängigkeit vom Meßmittelwert

(x) dargestellt sind, erkennen läßt, kann man in erster Nähenung für den gesamten untersuchten Konzentrationsbereich einen linearen Zusammenhang unterstellen. Regressionsrechnerisch erhält man für den Achsenabschnitt a $=0,09 \pm 0,081 \mu \mathrm{g} / \mathrm{l}$, was einen absoluten Fehler unwahrscheinlich macht, und fur die Steilheit $b=0,07 \pm 0,007$, was einem gewichteten mittleren relativen Fehler von $7 \%$ entspricht.

\subsubsection{Bestimmungs- und Nachweisgrenze}

Die untere Bestimmungsgrenze - in strikter Definition diejenige Konzentration, die noch mit Variationskoeffizienten $\leqslant 10 \%$ bestimmt werden kann - liegt nach den oben besprochenen Daten für die beschriebene Methode bei Einsatz von $2 \mathrm{ml}$ Probe zwischen 22 und $55 \mu \mathrm{g} / \mathrm{l}$. Zieht man allerdings in Betracht, daß es sich hier um eine im niedrigen Konzentrationsbereich recht heikle direkte Isotopenverdünnungsmethode handelt, und nimmt man Variationskoeffizienten bis $15 \%$ in Kauf, so zeigen die vorgelegten Werte, daß bis hinab zu $11 \mu \mathrm{g} / \mathrm{l}$ verläßlich bestimmt werden kann.

Aus den Ergebnissen mit Humanplasmen, denen lediglich zur Standardisicrung ${ }^{14} \mathrm{C}$-markiertes Propafenon zugesetzt worden war, errechnet sich anhand der dabei erhaltenen Werte $(-0,33 \pm 1,05 \mu \mathrm{g} / \mathrm{l} ; \mathrm{vgl}$. Tab. 4) die untere

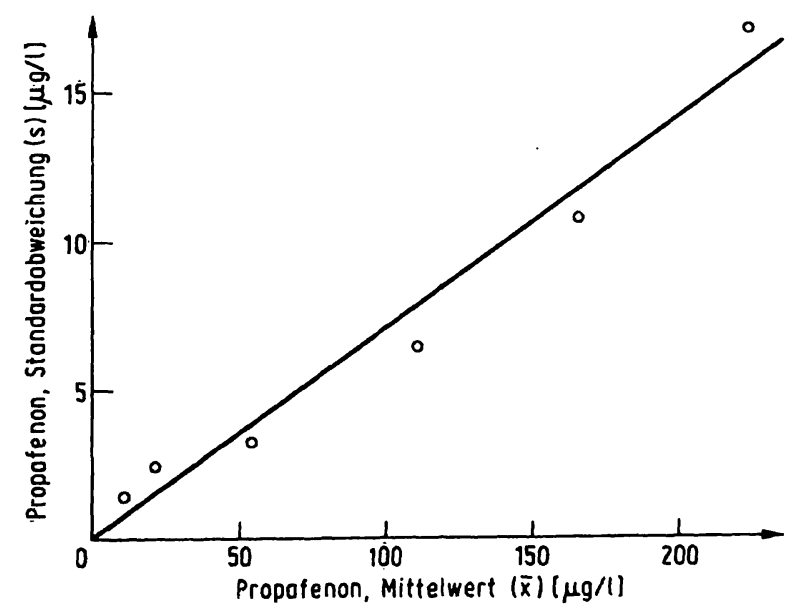

Abb. 2. Propafenon-Methodenprüfung (direktc Isotopenverdünnung mit Peakflächenmessung).

Fehlerstruktur.

Abhängigkeit der Standardabweichung vom Melsmittelwert. 
Tab. 4. Propafenon-Methodenprüfung (direkte Isotopenverdünnung nit Peakflächenmessung).

Lirgebnisse mit Leerplasmen zur Ermittlung der Nachweisgrenze.

\begin{tabular}{|c|c|c|}
\hline Probe & $\begin{array}{l}\text { Scheinbare Propafenon- } \\
\text { Konzentration } \\
(\mu \mathrm{g} / \mathrm{l})\end{array}$ & - \\
\hline $\begin{array}{l}1 \\
2 \\
3 \\
4 \\
5 \\
6 \\
7 \\
8 \\
9 \\
10 \\
11 \\
12 \\
13 \\
14 \\
15 \\
16\end{array}$ & $\begin{array}{r}-1,59 \\
-1,69 \\
-0,08 \\
-2,38 \\
-1,31 \\
-0,08 \\
-0,59 \\
0,98 \\
1,71 \\
0,38 \\
0,35 \\
0,15 \\
0,81 \\
0,39 \\
-0,19 \\
-0,23\end{array}$ & $\begin{array}{l}\bar{x}=-0,33 \mu \mathrm{g} / 1 \\
\mathrm{~s}_{\mathbf{x}}=1,05 \mu \mathrm{g} / 1 \\
\mathrm{~s}_{\bar{x}}=0,26 \mu \mathrm{g} / 1\end{array}$ \\
\hline
\end{tabular}

Nachweisgrenze - definiert als das Dreifache der Standardabweichung über dem Meßmittelwert - zu etwa $3,5 \mu \mathrm{g} / \mathrm{l}$.

\subsection{Peakhöhen als Meßparameter}

\subsubsection{Richtigkeit}

Die in Tabelle 5 zur Richtigkeit der Methode zusammengestellten Daten zeigen Wiederfindungsquoten zwischen 96 und 100\%, die in keinem Fall signifikant von $100 \%$ abweichen. Hinsichtlich möglicher Gründe für die in der Tendenz zu niedrigen Werten sei auf die oben (3.1.1) ausgesprochene Vermutung eines systematischen Fehlers beim Ansatz der spezifischen Aktivität des Tracers hingewiesen.

Insgesamt gesehen bestätigt eine Korrelationsanalyse zwischen gegebenen und gefundenen Werten anhand von Einzeldaten, daß das Analysenverfahren mit der hier geübten Standardisierung richtige Werte liefert, wie der Regressionskoeffizient $b=0,994 \pm 0,006$ zeigt.

Demgemäß ist auch bei den verschiedenen in der gleichen Serie analysierten Konżentrationen die strikte Proportionalität gewährleistet, wie die Abbildung 3a und 3b für die Serien mit den höchsten und niedrigsten Korrelationskoeffizienten zeigen.

$\cdot 1$

\subsubsection{Präzision}

Die Angaben zur Präzision des Gesamtverfahrens bei jeder der vorgegebenen Konzentrationen sind ebenfalls der Tabelle 5 zu entnehmen. Die Variationskoeffizienten fallen von etwa $11,4 \%$ bei $11 \mu \mathrm{g} / \mathrm{l}$ auf etwa $2-4 \%$ bei den höheren Konzentrationen.

Wie aus Tabelle 6 hervorgeht, ist auch hier die Reproduzierbarkeit des Verfahrens von Tag zu Tag nahëzu identisch mit der des Gesamtverfáhrens, unabhängig davon, ob Unikate oder Duplikătè analysiert werdẹn.

Die Präzision der Duplikatbestimmungen ist in Tabelle 7 verzeichnet. Die durchschnittlichen Variationskoeffizienten schwanken - unabhängig von der Sollkonzentration - zwischen 1,5 und 3,5\%, höhere Variationskoeffizienten als $10 \%$ werden in keinem Fall beobachtet.

Wenn man bei der Anwendung der Methode im Routinebetrieb gerade noch einen Variationskoeffizienten von $10 \%$ bei den D̄oppelbestimmungen akżeptiert - das entspricht einem Verhältnis von $1: 1,15$ bei den Einzelbestimmungen -, so hat man nach diesen Datẹn nư gelegentlich Ausfälle zu erwarten, die eine Wiederholung der Ánalyse notwendig machen würden.

Die Gefahr einer Verfälschung der Aussage aufgrund von nicht eliminierten Ausreißern ist bei Berücksichtigung dieser bei der Routineanwendung einer Reihe von analytischen Methoden bewährten Vorgehenswweise jedoch minimiert.

Wie Abbildung 4 zeigt, kann man in erster Näherung einen linearen Zusammenhang zwischen Standardabweichungen und Meßmittelwert über den gạnzen

Tab. 5. Propafenon-Methodenprüfung (direkte Isotopenverdünnung mit Peakhöhenmessung). Angaben zu Richtigkeit und Präzision, Mittelwert der Dụplikatanalysen als Meßwert.

\begin{tabular}{|c|c|c|c|c|c|c|}
\hline \multirow[b]{2}{*}{$\begin{array}{l}\text { Vorgabe- } \\
\text { konzentration } \\
\text { gegeben } \\
(\mu \mathrm{g} / \mathrm{l})\end{array}$} & \multirow[b]{2}{*}{$\begin{array}{l}\text { Mittelwert } \\
\text { gefunden } \\
(\mu \mathrm{g} / 1)\end{array}$} & \multirow[b]{2}{*}{$\mathrm{n}$} & \multirow{2}{*}{$\begin{array}{l}\text { Richtigkeit } \\
\text { gefunden / gegeben } \\
(\%)\end{array}$} & \multirow[b]{2}{*}{$\begin{array}{l}\text { gefunden - gegeben } \\
(\mu \mathrm{g} / \mathrm{l})\end{array}$} & \multicolumn{2}{|c|}{ Präzision der Methode } \\
\hline & & & & & $\begin{array}{l}\text { Standard- } \\
\text { abwei- } \\
\text { chung } \\
(\mu \mathrm{g} / \mathrm{l})\end{array}$ & $\begin{array}{l}\text { Variations- } \\
\text { koeffi- } \\
\text { zient } \\
(\%)\end{array}$ \\
\hline $\begin{array}{r}11,17 \\
22,34 \\
55,53 \\
110,10 \\
167,51 \\
221,38\end{array}$ & $\begin{array}{r}10,78 \pm 0,43 \\
21,83 \pm 0,44 \\
53,31 \pm 0,80 \\
107,48 \pm 0,97 \\
164,27 \pm 0,96 \\
221,08 \pm 2,67\end{array}$ & $\begin{array}{l}8 \\
8 \\
8 \\
8 \\
8 \\
8\end{array}$ & $\begin{array}{l}96,49 \pm 3,88 \\
97,72 \pm 1,96 \\
96,00 \pm 1,43 \\
97,61 \pm 0,88 \\
98,06 \pm 0,57 \\
99,86 \pm 1,20\end{array}$ & $\begin{array}{l}-0,38 \pm 0,43 \\
-0,50 \pm 0,44 \\
-2,21 \pm 0,80 \\
-2,61 \pm 0,97 \\
-3,23 \pm 0,96 \\
-0,29 \pm 2,67\end{array}$ & $\begin{array}{l}1,23 \\
1,24 \\
2,26 \\
2,76 \\
2,71 \\
7,56\end{array}$ & $\begin{array}{r}11,39 \\
5,67 \\
\therefore 4,23 \\
2,56 \\
1,65 \\
3,42\end{array}$ \\
\hline
\end{tabular}




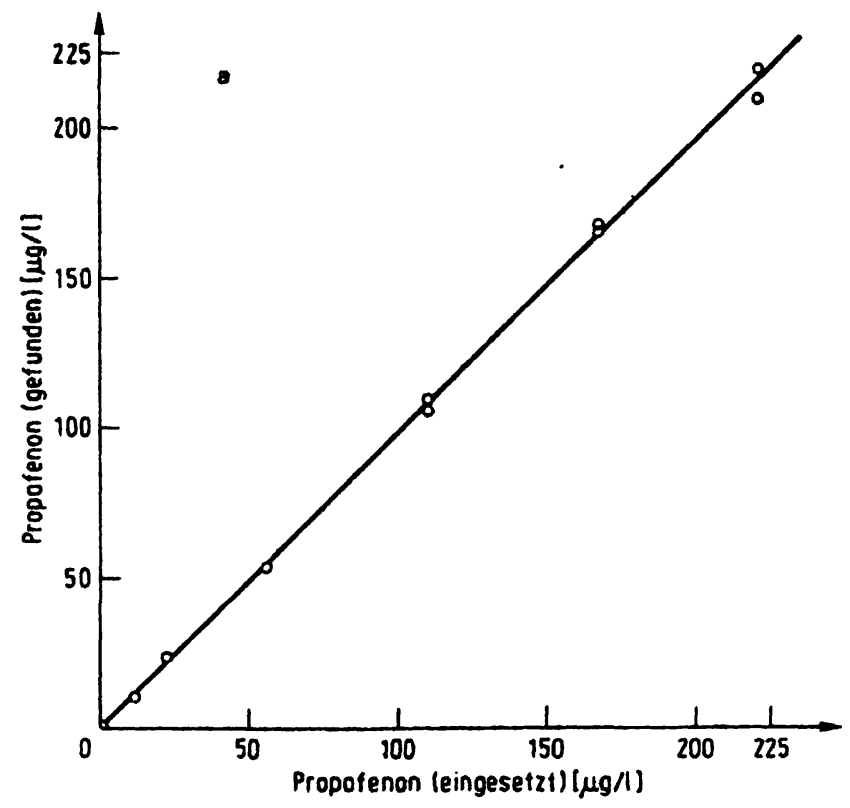

Tab. 6. Propafenon-Methodenprüfung (direkte Isotopenverdünnung mit Peakhöhenmessung).

Interserienstreuung.

\begin{tabular}{|c|c|c|c|c|}
\hline \multirow{3}{*}{$\begin{array}{l}\begin{array}{l}\text { Vorgabe- } \\
\text { konzentra- } \\
\text { tion }\end{array} \\
(\mu \mathrm{g} / 1)\end{array}$} & \multicolumn{2}{|c|}{$\begin{array}{l}\text { Variations- } \\
\text { koeffizienten } \\
\text { bci Analysen } \\
\text { von Unikaten }\end{array}$} & \multirow[b]{3}{*}{$\overline{\mathrm{VK}}(\%)$} & \multirow{3}{*}{$\begin{array}{l}\text { Variations- } \\
\text { koeffizienten } \\
\text { bei Analysen } \\
\text { von Duplikaten } \\
\text { VK (\%) }\end{array}$} \\
\hline & $\begin{array}{l}\text { 1. Wert } \\
\text { 2. Wert }\end{array}$ & & & \\
\hline & VK (\%) & $\mathrm{n}$ & & \\
\hline 11,1700 & $\begin{array}{l}12,77 \\
11,02\end{array}$ & $\begin{array}{l}8 \\
7\end{array}$ & 11,95 & 11,39 \\
\hline 22.3400 & $\begin{array}{l}5,49 \\
7.08\end{array}$ & $\begin{array}{l}8 \\
7\end{array}$ & 6,23 & 5,67 \\
\hline 55.5300 & $\begin{array}{l}5,96 \\
3.90\end{array}$ & $\begin{array}{l}8 \\
8\end{array}$ & 4,93 & 4,23 \\
\hline 110,1000 & $\begin{array}{l}4,16 \\
2.75\end{array}$ & $\begin{array}{l}8 \\
8\end{array}$ & 3,46 & 2,56 \\
\hline 167,5100 & $\begin{array}{l}1.78 \\
2,73\end{array}$ & $\begin{array}{l}8 \\
8\end{array}$ & 2,25 & 1,65 \\
\hline 221,3800 & $\begin{array}{l}4,64 \\
3,06\end{array}$ & $\begin{array}{l}8 \\
8\end{array}$ & 3.85 & 3,42 \\
\hline
\end{tabular}

Tab. 7. Propafenon-Methodenprüfung (direkte Isotopenverdünnung mit Peakhöhenmessung).

Präzision von Duplikaten (Variationskoeffizienten).

$\begin{array}{lllllll}\text { Vorgabekonzentra- } \quad 11,2 & 22,3 & 55,5 & 110,1 & 167,5 & 221,4\end{array}$ tionen $(\mu \mathrm{g} / \mathrm{l})$

\begin{tabular}{lcccccc}
\hline Serie 1 & 0,8 & 0,4 & 1,0 & 2,8 & 5,2 & 1,5 \\
Serie 2 & 4,7 & 5,9 & 0,1 & 4,9 & 1,1 & 4,5 \\
Serie 3 & 0,8 & 2,1 & 3,8 & 0,4 & 0,4 & 0,4 \\
Serie 4 & 1,6 & 1,4 & 1,0 & 2.3 & 1,0 & 3,1 \\
Serie 5 & 4,6 & 4,2 & 2,7 & 9,7 & 0,1 & 2,1 \\
Serie 6 & 7,7 & 4,5 & 9,6 & 4,7 & 0,8 & 4,6 \\
Serie 7 & 4,6 & 1,3 & 0,1 & 1,3 & 0,7 & 1,0 \\
Serie 8 & - & - & 1,8 & 1,4 & 2,9 & 3,0 \\
Mittelwert: & 3,1 & 2,5 & 2,5 & 3,5 & 1,5 & 2,5 \\
\hline
\end{tabular}

-: keine Duplikate vorhanden

Abb. 3a und 3b. Propafenon-Methodenprüfung (direkte Isotopenverdünnung mit Peakflächenmessung).

a) Soll/1st-Vergleich Serie $4(r=0,999)$

b) Soll/lst-Vergleich Serie 7 ( $\mathrm{r}=0,998)$

Abb. 4. Propafenon-Methodenprüfung (direkte Isotopenverdünnung mit Peakhöhenmessung).

Fehlerstruktur.

Abhängigkeit der Standardabweichung vom Meßmittelwert.

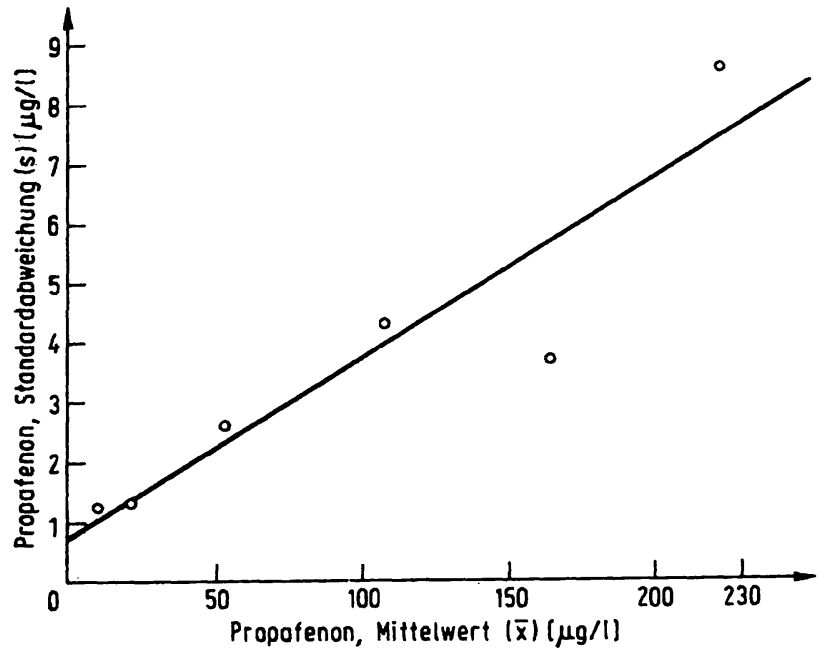

J. Clin. Chem. Clin. Biochem. / Vol. 20, 1982 / No. 1 
untersuchten Konzentrationsbereich unterstellen. Aus der Regressionsanalyse ergibt sich mit $a=0,73 \pm 0,79$ $\mu \mathrm{g} / \mathrm{l}$ das Fehlen eincs absoluten Fehlers; der mittlere gewichtete relative Fehler läßt sich zu etwa 3,5\% abschätzen. Wegen der in dem geprüften Konzentrationsbereich annähernd konstanten Variationskoeffizienten kann die lineare Funktion $s=0,73+0,03 \cdot \bar{x}$ zur Gewichtung bei Anpassungsrechnungen Verwendung finden, um die notwendige Voraussetzung homogener Varianzen zu gewährleisten.

\subsubsection{Bestimmungs- und Nachweisgrenze}

Nach den Werten der Tabelle 5 sind als untere Bestimmungsgrenze bei der Anwendung dieser Methode mit Peakhöhenmessung etwa $10 \mu \mathrm{g} / 1$ anzusetzen.

Die untere Nachweisgrenze errechnet sich aus den in Tabelle 8 angegebenen Ergebnissen der Analyse von Humanleerplasmen, denen lediglich zur Standardisierung ${ }^{14} \mathrm{C}$-Propafenon zugesetzt worden war, zu etwa $2,5 \mu \mathrm{g} / \mathrm{l}$.

\subsection{Schlußfolgerungen}

Die hier vorgelegten Daten zeigen, daß unter den gegebenen Umständen das Prinzip der direkten Isotopenverdünnung für die Bestimmung von Propafenon genutzt werden kann.

Wie aus einem Vergleich der Tabellen 1 und 5 sowie 2 und 6 hervorgeht, ist dabei der Quantifizierung durch Peakhöhenmessung der Vorzug zu geben, da die Reproduzierbarkeit deutlich besser ist (etwa Faktor 2). Dieses Resultat ist auf den ersten Blick überraschend, da normalerweise die Peakfläche als gegenüber den Irregularitäten chromatographischer Verfahren (Peakform, Retentionszeit) robuster Parameter anzusehen ist.

Tab. 8. Propafenon-Methodenprüfung (direkte Isotopenverdünnung mit Peakhöhenmessung).

Ergebnisse mit Leerplasmen zur Ermittlung der Nachweisgrenze.

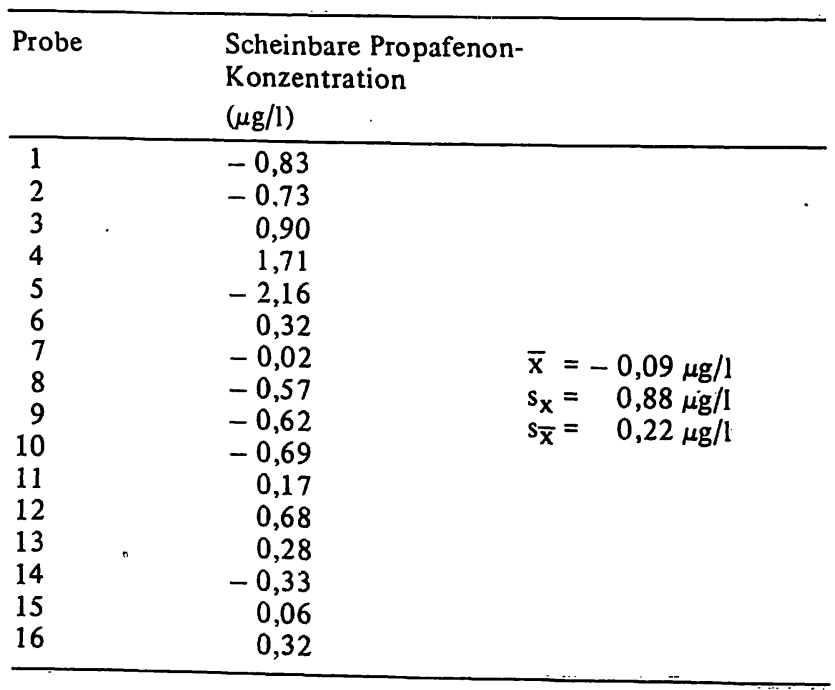

Eine detaillierte Betrachtung der den Ergebnissen zugrundeliegenden Rohdaten läßt jedoch erkennen, daß besonders bei Analysen komplexer biologischer Matrizen im hochempfindlichen Bereich die exakte Ermittlung der für die Peakauswertung verwendeten Grundlinie der problematischste Schritt ist.

Auch wenn durch den Einsatz eines Rechnerprogramms (s. 2.6) jegliche bei manueller Durchfürung dieser Operation mögliche Willkürr vermieden wird, ist auch bei Anwendung eines Algorithmus die Lage der Grundlinie zwar reproduzierbar, jedoch von der Umgebung des Peaks nicht unabhängig. Die sich damit zwangsläufig auch bei Wiederholungsmessungen ergebenden Unterschiede bei der Grundlinie wirken sich im niedrigen Konzentrationsbereich verständlicherweise auf die Höhenmenessung wèit weniger aus als auf die Flächenmessung, was die bessere Reproduzierbarkeit der mit der Peakhöhenmessung gewonnenen Ergebnisse erklärt.

Die Leistungsfähigkeit dieser direkten Isotopenverdünnungsmethode ist einmal dadurch beschränkt, däß ihr eigentliches Meßergebnis als Differenz (Gèsamtmenge Standardmenge) bezüglich seiner Präzision direkt von der eingesetzten Standardmenge abhängt, die wiederum von der spezifischen Radioaktivität des Tracers bestimmt wird. Die andere, für den speziellen Fall wesentlichere Limitierung liegt in der Empfindlichkeit und Güte der Bestimmungsmethode als solcher begründet. Hier deutet sich nach unseren früheren Untersuchungen (11) die Möglichkeit an, unter Wechsel der chromatogräphischen Technik (reverse phase HPLC) bei einem Kompromiß zwischen Ausbeute bei der Probenreinigung und der Reinheit des Extraktes durch Messung im kürzerwelligen UV zu einer nochmaligen Empfindlichkeitssteigerung zu gelangen. $\mathrm{Ob}$ dies jedoch bei der direkten Isotopenverdünnung zum Tragen kommt, muß erst geprüft werden.

Da die hier geprüfte direkte Isotopenverdünnungsmethode weitestgehend unter den gleichen experimentellen Bedingungen wie das früher beschriebene Ver: fahren mit interner Analogstandardisierung durchgeführt wurde, ist ein aussagekräftiger Vergleich der Leistungsfähigkeit beider Methoden möglich

Dabei zeigt sich, daß unterhalb einer Konzentration von $20 \mu \mathrm{g} / \mathrm{l}$ und damit vor allem auch bei der unteren Bestimmungsgrenze sich die Nachteile der direkten Isotopenverdünnung bemerkbar machen, die einmal in ihrer Eigenșchaft als Differenzmethode und zum anderen in der fehlenden Standardisierung bei der Chromatographie begründet sind. Wie oben bereits diskutiert, kann der erste Nachteil durch Verwendung eines Tracers mit höherer speżifischer Radioaktivität minimięrt werden; der zweite muß̣ zwar prinzipiell in Kauf genommen werden, er wird jedöch im Lauf der apparativen Weiterentwicklung immer weniger ins Gewicht fallen. 
Im Konzentrationsbereich ab $20 \mu \mathrm{g} / \mathrm{l}$ sind beide Methoden hinsichtlich Präzision und Richtigkeit gleichwertig, wie ein Vergleich der in Tabelle 5 verzeichneten Charakterisierungsdaten für die direkte Isotopenverdünnung mit den entsprechenden Werten für die interne Analogstandardisierung erkennen läßt.

Wenn keine größeren Ansprüche an die Empfindlichkeit der Analytik gestellt zu werden brauchen, kann für bestimmte Untersuchungen ein allgemeiner Vorteil der

\section{Literatur}

1. Brown, P. R. (1973) "High pressure liquid chromatography: Biochemical and biomedical application". Academic Press, New York and London.

2. Burchfield, H. P. \& Storrs, E. E. (1970) "Biochemical applications of gas chromatography". Academic Press, New York and London.

3. Rittenberg, D. \& Foster, G. L. (1940) J. Biol. Chem. 133, 737-744.

4. Gaffney, T. E., Hammar, C. G., Holmstedt, B. \& McMahon, R. E. (1971) Anal. Chem. 43, 307-310.

5. Cho, A. K., Lindeke, B., Hodshon, B. J. \& Jenden, D. J. (1973) Anal. Chem. 45, 570-574.

6. Klcin, E. R. \& Klein, P. D. (1975) Proceedings of the Second International Congress of Stable Isotopes. Argonne National Laboratory, Argonne.

7. Björkhem, I., Blomstrand, R., Lautto, O., Scensson, L. \& Öhmann, G. (1976) Clin. Chem. 22, 1789-1801. direkten Isotopenverdünnung ausschlaggebend werden, der die interne Standardisierung jeder einzelnen Probe direkt nach ihrer Gewinnung erlaubt.

\section{Danksagung}

Ich danke Frau Ursula Kripp für ihre sorgfältige und exakte technische Mitarbeit.

8. Klein, E. R. \& Klein, P. D. (1978) Biomed. Mass Spectrom. $5,91-92$.

9. Baba, S., Goromarn, T., Yamazaki, K. \& Kasnya, Y. (1980) J. Pharm. Sc. 69, 1300-1307 (dort weitere Literatur).

10. Schütte, H. R. (1966) Radioaktive Isotope in der organischen Chemie und Biochemic. Verlag Chemie, Weinheim.

11. Brode, E., Sachse, R. \& Hoffmann, H. D. (1982) Arzneim.Forsch./Drug Res. 32 (I) Nr. 1.

12. Cacsar, F. \& Klicr, M. (1974) Chromatographia 7, 526-529.

13. Diem, K. \& Lentner, C. (Hrsg.) (1968) „Documenta Geigy: Wissenschaftliche Tabcllen". 7. Auflage. J. R. Geigy AG, Pharma, Basel. S. 173-174.

14. Diem, K. \& Lentner, C. (Hrsg.) (1968) „Documenta Geigy: Wissenschaftliche Tabellen". 7. Auflage. J. R. Geigy AG, Pharma, Bascl. S. 175-180, S. 180-181.

Dr. E. Brode

BASF Aktiengesellschaft

Exp.-Med. Forschung u. Entwicklung

Dept. Biochemie

D-6700 Ludwigshafen 
